\title{
Les contes de la plume blanche
}

\author{
Anne-Laurence Fitère \\ Illustrations d'Anja Klauss \\ Enregistrements de Rebecca Hampton et Serge Dupire \\ Le Cherche-Midi, 2013
}

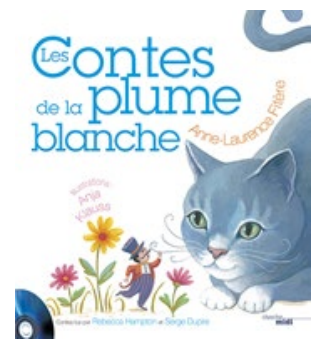

Anne-Laurence Fitère est morte il y a peu de temps, elle avait 40 ans. La Maison du cancer a pris le deuil. Cette journée du 8 octobre 2012 avait vu disparaître son hôtesse la plus fervente et la plus dynamique. Créatrice de ce site Internet original, Anne-Laurence Fitère, journaliste pendant dix ans aux Échos, était une jeune femme complète et créative comme l'est le livre qu'elle nous laisse. Ce livre est (évidemment) un livre de contes. Un livre qui, comme son site, utilise plusieurs médias, le texte, les dessins colorés, les voix chaleureuses et la musique.

Anne-Laurence Fitère a dédié son livre tout particulièrement aux enfants malades. Elle y déploie avec sensibilité des histoires teintées de modernité, mais qui revisitent les grands mythes : la Tour de Babel, les hommes devenus animaux, les rêves qui permettent de communiquer, l'Éden, l'espace habité par des puissances égocentriques. Chaque histoire est un enchantement, grâce aux voix de Rebecca Hampton et Serge Dupire qui s'en donnent à cœur joie dans les bruitages, mais aussi par l'introduction des sons des animaux, ce qui permet à un enfant hospitalisé de se croire dans la jungle ou dans la savane. Souvent, le soir à l'hôpital, quand les lumières s'éteignent et que les enfants fatigués ne peuvent plus regarder la télévision ou l'ordinateur, écouter les contes d'Anne-Laurence Fitère leur permet de se reposer tout en laissant errer leur imaginaire.

C'est un peu un livre à tout faire : on le feuillette distraitement pendant les longues attentes, on le regarde et on le recopie si l'on sait dessiner, on demande à ses parents de le lire et alors leur interprétation est un nouveau message ; enfin, on peut l'écouter pour les contes mais aussi pour les morceaux de musique. Les morceaux sont tous au piano et illustrent parfaitement les histoires. De plus, ils permettent de cultiver la mémoire parfois chahutée par les chimio- ou radiothérapies. Cette mémoire à court terme qui a besoin d'une plus grande concentration est stimulée, mais aussi la mémoire à long terme des enfants malades. Parfois, et les congrès scientifiques sur les adolescents et jeunes adultes anciens malades l'ont montré, la mémoire de l'enfance malade fait défaut ou encore, les souvenirs sont réprimés parce qu'ils font souffrir les parents... Avec le livre, et surtout les morceaux de musique, le souvenir sera à la fois mieux fixé, mais surtout plus doux à évoquer. Parents et enfants pourront ainsi plus agréablement se remémorer les temps difficiles et anxiogènes de la maladie.

Parmi les contes, on ne trouve pas d'histoire de maladie, mais plutôt des variations de taille, de corpulence, on l'aura compris une image du corps qui change, comme lors du cancer. Autre thématique : les échanges avec les animaux qui évoquent les moments de régression psychique pendant la maladie. Enfin, le langage qui permet d'échanger, mais aussi de faire semblant de ne pas se comprendre nous a évoqué les moments délicats de révélation des mauvaises nouvelles, souvent présentes hélas pendant le voyage au pays du cancer...

La bonne nouvelle, chère Anne-Laurence, c'est que vous avez laissé bien des choses de votre court passage sur terre, le site de la Maison du cancer et ce beau livre qui, sans conteste, donnera beaucoup à rêver et à imaginer aux enfants.

\author{
M.-F. Bacqué \\ Rédactrice en chef de Psycho-Oncologie \\ EA 3071, université de Strasbourg, \\ F-67000 Strasbourg, France \\ Correspondance : mfbacque@club-internet.fr
}

